\title{
RÉPARTITION TISSULAIRE ET SUBCELLULAIRE DU STRONTIUM CHEZ LE RAT $\left(^{*}\right)$
}

\author{
J. TINE, G. ROCQUET (**) \\ (Manuscrit reçu le 4 novembre 1974)
}

\begin{abstract}
RESUME
Après injection de ${ }^{85} \mathrm{Sr}$ à des rats, une part non négligeable de la nadioactivité se localise au niveau des tissus mous. Pour des temps de diffusion courts, le poumon, le rein, le foie et les testicules sont les plus chargés en cations. Avec des temps plus longs, on constate que la charge est maximale 48 heures après injection du ${ }^{85} \mathrm{Sr}$. On note également que l'hypophyse, la thyroïde et les surrénales concentrent des quantités importantes de strontium. Nous mettons également en évidence que, dans le parenchyme hépatique, la distribution du ${ }^{85} \mathrm{Sr}$ se fait essentiellement dans les mitochondries, microsomes et noyaux.
\end{abstract}

\begin{abstract}
After injection of ${ }^{85} \mathrm{Sr}$ to rats, a non negligible part of the radioactivity was located in soft tissues. For short times of diffusion, lung, kidney, liver and testicles were the most loaded with cations. With longer times, a maximum charge was observed 48 hours after injection of $85 \mathrm{Sr}$. Hypophysis, thyroid and adrenals concentrated very important quantities of strontium.

We also show that the distribution of ${ }^{85} \mathrm{Sr}$ in hepatic tissue occured especially in mitochondriae, microsomes and nuclei.
\end{abstract}

Parmi les alcalino-terreux, le calcium est sans conteste le plus important sur le plan biologique; le strontium toutefois, a pris depuis un certain nombre d'années un très grand intérêt du fait de ses propriétés radiotoxicologiques. Celles-ci sont dues à son caractère ostéotrope et à l'un de ses isotopes radioactifs, le strontium 90. C'est l'un des produits de fission les plus dangereux. Du fait de sa période effective (environ 17 ans pour l'os), il peut ainsi irradier l'os et la moelle osseuse, provoquant dans certaines circonstances des tumeurs malignes et des leucémies [33].

(*) Communication présentée au Séminaire de Radiologie sur le thème de la contamination interne, 18-20 mars 1974 (Paris), organisé par la Société Française de Radioprotection.

(**) Centre de Recherches du Service de Santé des Armées - Division de Radiochimie rue du Lieutenant Raoul-Batany, 92140 Clamart.

RADIOPROTECTION, VOL. 9 - $\mathrm{N}^{\circ} 3$ 
En dehors de ces considérations radiobiologiques, il a tout de même été bien établi par un certain nombre d'auteurs que le strontium est présent à des concentrations très faibles dans différents tissus ou organes de mammifères $[27,14,19,12,31]$. Les études sur le métabolisme du strontium, conduites avec différents isotopes tels que ${ }^{90} \mathrm{Sr},{ }^{89} \mathrm{Sr},{ }^{87 m} \mathrm{Sr},{ }^{85} \mathrm{Sr}$, ont surtout porté sur la rétention et le métabolisme osseux de ceux-ci.

Peu de recherches ont été consacrées à la fixation du cation par les différents organes. Les travaux de Luning et coll. [20], de BruEs et coll. $[4,5]$ ont montré que le strontium diffuse dans tout l'organisme des mammifères et que certains organes comme le foie ou les testicules concentrent le cation. Les recherches de MAYNARD et Cotzias [23], de THIERs et VALlée [22] sur la distribution tissulaire du manganèse et du calcium radioactifs dans les différentes fractions subcellulairres de foie de rat ont mis en évidence une affinité spécifique des mitochondries pour ces éléments. Le même phénomène a été mis en évidence par VASINGTON en 1966 [32] et CARAFOLI en 1967 [6,9] avec un autre cation : le strontium.

Les travaux de CARAFOLI montrent qu'après une injection intrapéritonéale de ${ }^{85} \mathrm{Sr}$ celui-ci se concentre dans le foie très rapidement suivant en cela le calcium ; de plus, parmi les différentes fractions subcellulaires étudiées, les mitochondries et les microsomes sont les plus riches en cation.

Devant le peu de travaux consacrés à la distribution du strontium au niveau des tissus mous, nous nous proposons d'étudier la répartition du strontium 85 dans les organes et de vérifier que dans le foie, les organites subcellulaires captant le cation préférentiellement sont bien les mitochondries et les microsomes.

Nous étendrons cette étude à une fraction nouvelle, la fraction nucléaire.

\section{MATÉRIEL ET MÉTHODES}

\section{Distribution tissulaire du Strontium 85}

Les expérimentations ont été conduites avec du ${ }^{85} \mathrm{Sr}$, émetteur gamma.

Les observations faites par de nombreux auteurs concernant l'importance, sur le devenir métabolique de cet alcalino-terreux, de l'âge des animaux, de leur nourriture, du mode d'administration du radionucléide nous ont amenés à nous fixer des conditions expérimentales très strictes.

Une sélection sévère des animaux basée sur leur origine et sur leur taille a été effectuée (ainsi qu'un nombre important d'essais). Pour cela, nous avons choisi des rats mâles albinos de souche Wistar de 190 à $200 \mathrm{~g}$ provenant toujours du même élevage $\left({ }^{*}\right)$. Toutes les expérimentations ont été conduites avec des rats acclimatés au laboratoire pendant 8 jours et soumis toujours à la même nourriture.

(*) Sodelabo, Condé-sur-Huisne (Orne). 
Chaque animal reçoit par voie intraveineuse dans l'une des veines de la queue une dose de $5 \mu \mathrm{Ci}$ de ${ }^{85} \mathrm{Sr}$ en solution isotonique, pour $100 \mathrm{~g}$ de poids corporel. Après un temps déterminé, les animaux sont sacrifiés par section de la carotide. On prélève avec précaution et on pèse rapidement les organes étudiés en ayant eu soin d'éliminer au maximum le sang. Les différents prélèvements sont ensuite homogénéisés par chauffage à ébullition dans de la soude normale. Après refroidissement, on procède à la mesure de la radioactivité sur la totalité de l'échantillon à l'aide d'un scintillateur à INa $3^{\prime \prime} \times 3^{\prime \prime}$ à puits (Autogamma Packard).

\section{Préparation des fractions subcellulaires de foie de RAT}

Les noyaux, les mitochondries et les nicrosomes sont préparées par ultracentrifugation différentielle selon les tecl niques antérieurement décrites [30].

\section{RESULTATS ET DISCUSSION}

\section{RÉPARTITION DU STRONTIUM DANS LES ORGANES EN FONCTION DU TEMPS}

Nous avons effectué nos essais avec des quantités de ${ }^{85} \mathrm{Sr}$ exemptes d'entraîneur, c'est-à-dire sans strontium stable. Il a été démontré que, par

TABLEAU I

RADIOACTIVITÉ EXPRIMÉE EN PCi PAR MINUTE ET PAR Mg DE DIVERS ORGANES PRÉLEVÉS À DES TEMPS DIFFÉRENTS CHEZ DES RATS AYANT REÇU $5 \mu \mathrm{Ci}$ DE ${ }^{85} \mathrm{Sr}^{++}$TRACEUR POUR $100 \mathrm{~g}$ DE POIDS CORPOREL.

\begin{tabular}{lcccc}
\hline \multirow{2}{*}{ Organe } & \multicolumn{4}{c}{ Temps de prélèvement en minutes } \\
& 5 & 10 & 30 & 60 \\
\hline Cerveau & 1,3 & 2,2 & 2,6 & 3 \\
Coeur & 44 & 52,5 & 28,3 & 12,5 \\
Foie & 36,2 & 44,5 & 50,3 & 52,8 \\
Hypophyse & 8,7 & 12,8 & 20,2 & 22,4 \\
Poumon & 86,4 & 92 & 101 & 114,3 \\
Rate & 45,2 & 48,4 & 52,6 & 54,5 \\
Rein & 71,2 & 88,4 & 87,5 & 85,4 \\
Surrénales & 6,2 & 25 & 32 & 34 \\
Testicules & 27,5 & 32,4 & 39,6 & 41,2 \\
Thyrołde & 10,5 & 11,5 & 18,2 & 19,4 \\
Plasma & 15 & 8 & 5 & 3 \\
& & & & \\
\hline
\end{tabular}

vol. $9-\mathrm{N}^{\circ} 3$ 


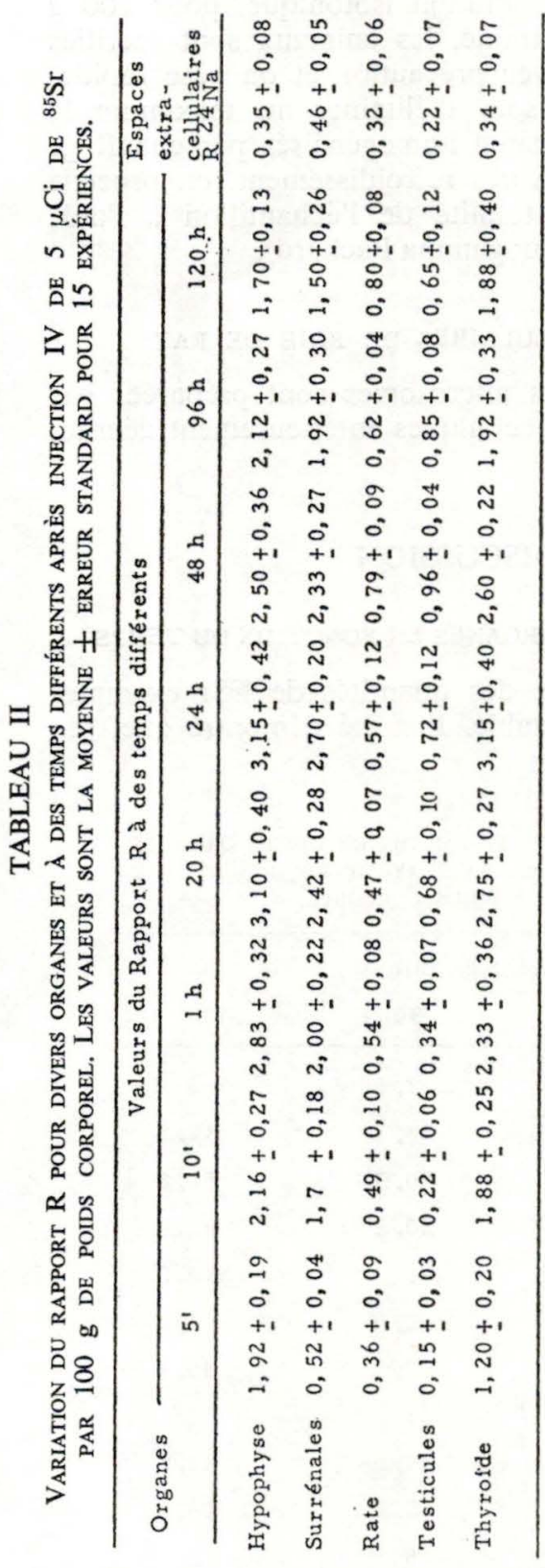

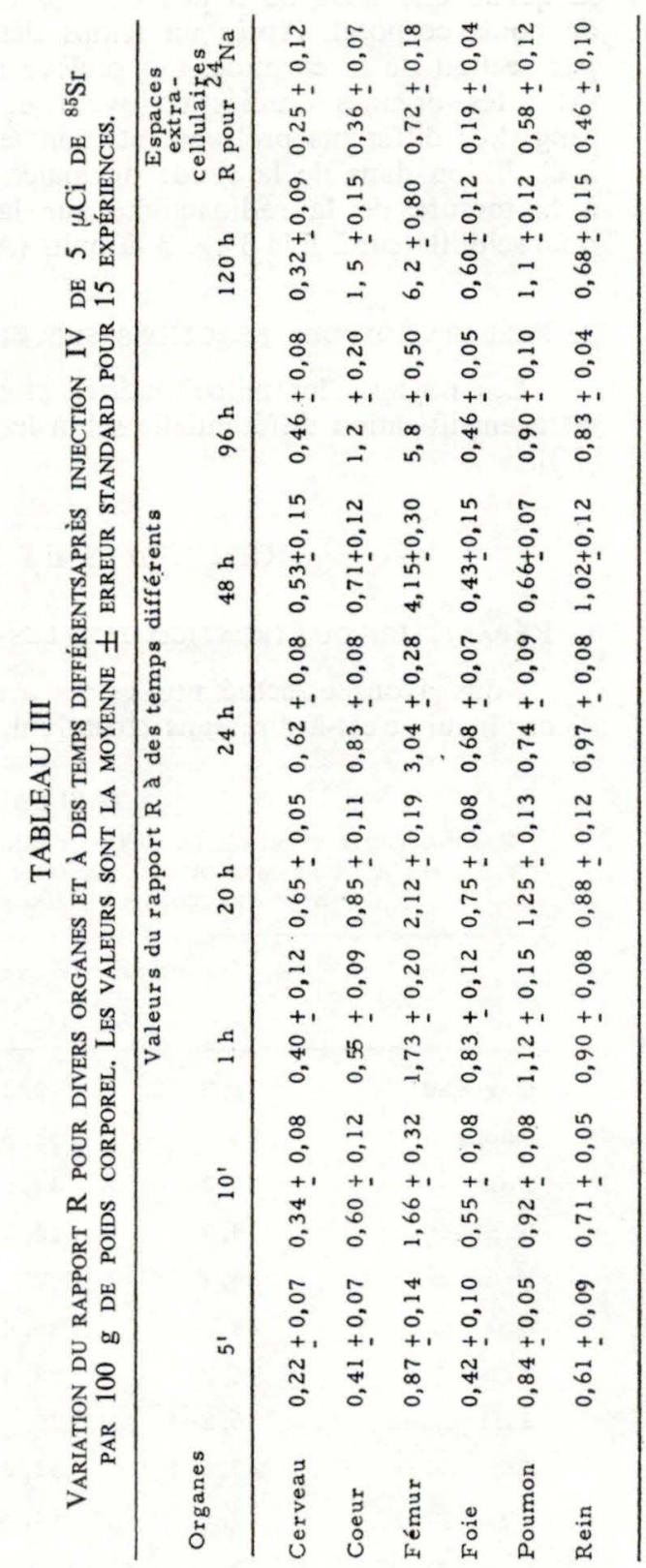


Injection intraveineuse ou intrapéritonéale, celui-ci diminue la charge de l'organisme $[10,11,26,28]$.

Une première série d'essais a été effectuée en prélevant les organes $5,10,30$ et $60 \mathrm{mn}$ après injection de ${ }^{85} \mathrm{Sr}$. Les résultats font l'objet du tableau I. De cette expérience il apparaît que le strontium diffuse rapidement dans les organes, en particulier, dans le poumon, le rein, la rate, le foie et les testicules, le cerveau étant le moins riche.

Après $5 \mathrm{mn}$, une grande partie de la radioactivité reflue du sang vers l'espace extravasculaire. Ce fait ayant déjà été signalé par LLYod [18] et BISCHOP et coll. [3], qui ont remarqué que le ${ }^{90} \mathrm{Sr}$ quitte rapidement chez le lapin le milieu interstitiel pour disparaître presque complètement en $2 \mathrm{~h}$ vers l'espace extracellulaire.

Nous voyons, en outre, que les testicules concentrent rapidement le ${ }^{85} \mathrm{Sr}$. Ce fait est à rapprocher des résultats trouvés par BRUEs et coll. [5] sur des souris mâles. Par ailleurs, ABERG et Gillner [1] ont montré que le ${ }^{89} \mathrm{Sr}$ diffuse au niveau de l'épididyme et du spermatozoïde pour se fixer préférentiellement sur les acides nucléiques pouvant ainsi provoquer des mutations génétiques.

L'organe le plus chargé en strontium 85 semble être le poumon: cela est certainement dû à la rétention rapide du cation dans le tissu cartilagineux des bronches, le strontium étant avant tout un élément à tropisme osseux tout comme le calcium $[2,13,17]$.

L'étude de la captation du cation a été poursuivie sur des expériences de plus longue durée. Nous nous sommes assurés au préalable que les déterminations de radioactivité restaient suffisantes pour être démonstratives. A cet effet, nous avons fait des mesures de ${ }^{85} \mathrm{Sr}^{++}$sur les organes prélevés $120 \mathrm{~h}$ après l'injection.

Les résultats obtenus sont significatifs, tous les comptages restant toujours supérieurs au double du bruit de fond. Nos recherches ont porté sur des temps allant de $5 \mathrm{mn}$ à $120 \mathrm{~h}$.

Pour faciliter l'interprétation des résultats, nous comparons sur les tableaux II et III, les rapports :

$$
\mathbf{R}=\frac{\text { Radioactivité d'un mg d'organe }}{\text { Radioactivité d'un mg de plasma }}
$$

qui permettent de juger de la fixation tissulaire du strontium.

On pourra parler d'une affinité élective $\mathrm{du} \mathrm{Sr}^{++}$pour un organe si le rapport précédent prend une valeur supérieure à celle des espaces extracellulaires de l'organe considéré [22]. Nous avons donc tout d'abord déterminé la valeur des espaces extracellulaires en calculant le même rapport $R$, après administration à l'animal de ${ }^{24} \mathrm{Na}$ dont on sait qu'il se répartit rapidement et uniformément dans le plasma et le liquide interstitiel sans pénétrer dans les cellules [21]. Les valeurs trouvées sont rapportées dans les tableaux 
II et III, le temps de diffusion du radionucléide étant de $2 \mathrm{~h}$. Nos résultats sont en accord avec ceux décrits dans la littérature [21, 22, 25].

Les valeurs des rapports $R$ comparées à celles des espaces extracellulaires (tableaux II et III) indiquent que le strontium traverse très facilement les membranes cellulaires et diffuse dans tous les organes. En outre, la charge en strontium dans les différents organes est maximale en $48 \mathrm{~h}$ pour décroître ensuite. Seul l'os continue à concentrer le cation après ce délai. Il faut également relever que l'hypophyse et la thyroïde concentrent des quantités importantes de strontium.

\section{Distribution SUbCellulaire DU STrontium 85 Dans Le tissu HÉPATIQUE}

Ayant mis à nouveau en évidence que le foie de rat est capable de capter du strontium après injection intraveineuse, nous nous sommes proposés de vérifier les travaux de CARAFOlI $[6,9,8]$ concernant la distribution de cet élément dans les subparticules de la cellule hépatique tout en complétant son travail par une étude de la fixation nucléaire du $\mathrm{Sr}^{++}$.

\subsection{Conditions expérimentales}

Le mode d'administration du radionucléide est identique à celui que nous avons décrit précédemment. La fraction que nous appelons «microsomes lourds » est celle recueillie après centrifugation à $30000 \mathrm{~g}$ pendant $20 \mathrm{mn}$. Le résidu est la fraction correspondant aux particules de faible densité restant à la surface lors de l'isolement des noyaux [30].

Le surnageant correspond à la somme des différents surnageants obtenus lors du lavage des microsomes [30].

\subsection{Résultats}

Le tableau 4 montre la distribution en pourcentage du ${ }^{85} \mathrm{Sr}^{++}$dans différentes fractions subcellulaires, les rats étant sacrifiés $5 \mathrm{mn}$ après injection intraveineuse du cation. Il montre que la majeure partie de la radioactivité se trouve dans la fraction mitochondriale et microsomale; de plus, un pourcentage relativement important se localise dans la fraction nucléaire. L'ensemble de nos résultats est en accord avec ceux obtenus par CARAFOLI [9]; nous mettons en évidence que dans la fraction appelée * résidu » par cet auteur, ce sont principalement les noyaux qui concentrent le cation.

La figure 1, représentant la distribution $\mathrm{du}^{85} \mathrm{Sr}^{++}$dans les subparticules en fonction du temps, montre que la radioactivité croît rapidement dans les premières $15 \mathrm{mn}$, pour décroître rapidement par la suite. La radioactivité de la fraction nucléaire et celle des « microsomes lourds » reste relativement constante; quant aux mitochondries leur charge en strontium reste à un niveau nettement supérieur aux autres fractions. 


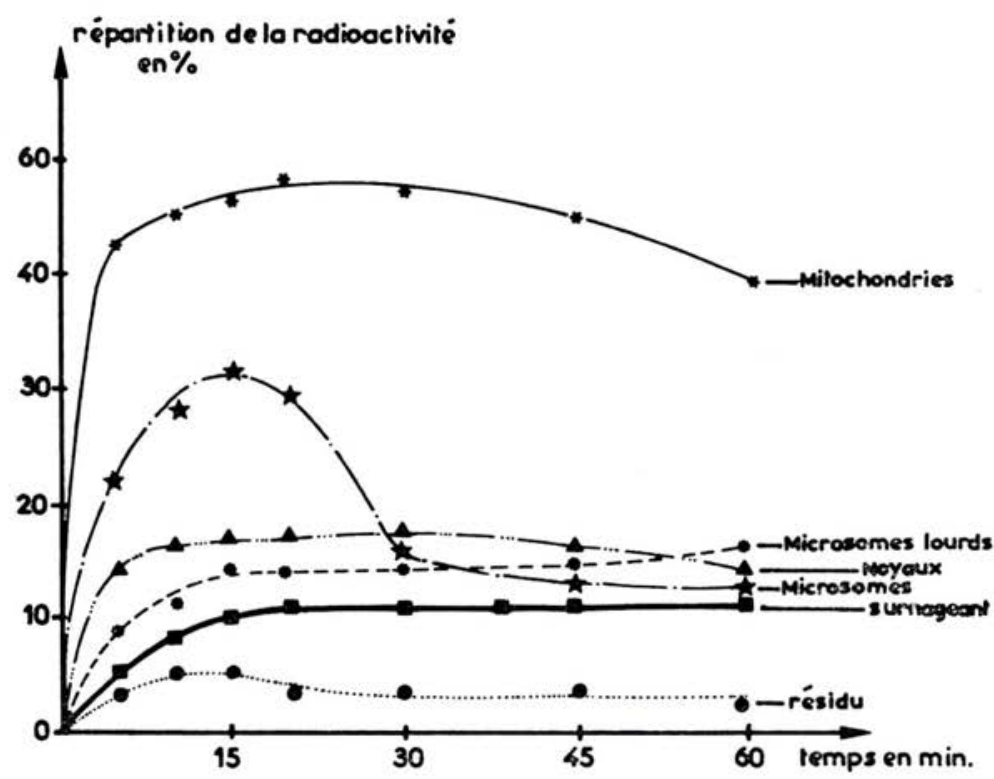

FIg. 1. - Distribution de la radioactivité dans différentes fractions subcellulaires de foie de rat à différents temps après injection de ${ }^{85} \mathrm{Sr}^{++}$

Dans le tableau 4, nous avons également porté les concentrations relatives de ${ }^{85} \mathrm{Sr}$ par $\mathrm{mg}$ de protéines, que nous avons comparées à la valeur obtenue pour l'homogénat non fractionné prise comme unité de référence. Les mitochondries sont les organites les plus chargés en ${ }^{85} \mathrm{Sr}$. Ceci est en accord avec les observations de MAYNARD et CotzIas [23] pour le manganèse, de CARAFoli $[6,7]$ pour le $\mathrm{Ca}^{++}$et $\mathrm{le} \mathrm{Sr}^{++}$, de Wester [35] et Cosmos [15] sur d'autres tissus tel que le cœur ou le muscle où là encore les mitochondries sont capables d'accumuler in vivo des cations bivalents.

\section{CONCLUSIONS}

Si la fixation du strontium au niveau osseux est largement décrite dans la littérature [16, 24, 34], par contre, celle qui se produit au niveau des tissus mous n'a fait l'objet à notre connaissance que de travaux peu nombreux.

La diffusion massive et rapide du strontium dans tous les organes étudiés constitue l'observation la plus frappante que nous ayons enregistrée au cours de nos essais. Ce phénomène est déjà net pour des temps courts, où l'on constate dès la $5^{\mathrm{e}} \mathrm{mn}$ qui suit l'injection, une radioactivité tissulaire supérieure à celle d'un même poids de plasma. Il apparaît donc que la

VoL. $9-\mathrm{N}^{\circ} 3$ 
membrane cellulaire est perméable au strontium, car celui-ci quitte très rapidement le compartiment vasculaire pour le milieu intracellulaire. L'étude du métabolisme du cation sur une période plus longue nous a permis d'observer que, dans les premières heures qui suivent son injection, le strontium se concentre surtout dans l'hypophyse, la thyroïde, les surrénales, le fémur et une fraction non négligeable au niveau des testicules. Cette activité spécifique élevée dans les glandes endocrines et particulièrement les glandes génitales avait déjà été observée dans notre laboratoire à la suite d'expérimentations sur des mammifères plus importants (travaux non publiés).

TABLEAU IV

Distribution SUBCELlULAIRE DU ${ }^{85} \mathrm{Sr}$.

LES RATS SONT SACRIFIÉS $5 \mathrm{mn}$ APRÈS L'INJECTION DU RADIOÉLÉMENT.

\begin{tabular}{lcc}
\hline Fraction & $\begin{array}{c}\text { dpm } \times \mathrm{mg}^{-1} \\
\text { de protélne }\end{array}$ & $\begin{array}{c}\text { distribution } \\
\text { en pourcentage }\end{array}$ \\
\hline Noyau & $0,74 \pm 0,025$ & $14,3 \pm 0,96$ \\
Résidu & $0,25 \pm 0,018$ & $3,5 \pm 0,75$ \\
Mitochondrie & $1,95 \pm 0,150$ & $46,5 \pm 3,25$ \\
Microsomes lourds & $1,20 \pm 0,110$ & $8,2 \pm 2,10$ \\
Microsomes & $1,02 \pm 0,06$ & $20,2 \pm 1,28$ \\
Surnageant & $0,32 \pm 0,034$ & $4,7 \pm 0,86$ \\
\hline
\end{tabular}

* Les valeurs sont exprimées en dpm par mg de protéine, en prenant COMME VALEUR DE RÉFÉRENCE CELLE DE L'HOMOGÉNAT ÉGALE À 1,00 .

LES RÉSULTATS SONT LA MOYENNE DE 10 DÉTERMINATIONS \pm ERREUR STANDARD.

En plus du risque dû à l'irradiation de la moelle osseuse, il semblerait donc nécessaire de prendre en considération l'irradiation de ces glandes endocrines, car elle pourrait se traduire par des troubles du métabolisme et un risque génétique. Ce risque génétique est d'autant plus important que, dans les études de localisation faites au niveau subcellulaire, nous avons mis en évidence, qu'en dehors d'organites tels que les microsomes et les mitochondries, le noyau de la cellule est capable de fixer des quantités non négligeables de strontium.

\section{REMERCIEMENTS}

Nous remercions MM. C. Duhamel et H. Monachon pour leur excellente aide technique. 


\section{BIBLIOGRAPHIE}

[1] Aberg B., Gillner M. In «Strontium Metabolism » J.M.A. Lenihan, J. Loutit, J.H. Martin, Eds. Academic Press, N.Y., p. 260 (1967).

[2] Bauer G.C.H., Carlsson A., Lindquist B. Acta Physiol. Scand., 35, 56, (1955).

[3] Bishop M., Harrison G.E., RAymond G.E., Sutton W.H.A., Rundo J. Int. J. Radiat. Biol., 2, 125, (1960).

[4] Brues A.M., Auerbach H., Deroche G. ANL-Report ANL 6823, 76-80, (1963).

[5] Brues A.M., Auerbach H., Deroche G. In «Strontium Metabolism » J.M.A. Lenihan, J.F. Loutit, Martin J.H., Eds. Academic Press, N.Y., p. 207, (1967).

[6] Carafoli E. Lo Sperimentale, 117, 19, (1967).

[7] Carafoli E. Lo Sperimentale, 117, 31, (1967).

[8] Carafoli E. Sper. Arch. Biol. Norm. Patol., 117, 19, (1967).

[9] Carafoli E. J. Gener. Physiol., 50, 1851, (1967).

[10] Carlquist B., Nelson A. Acta Radiol., 54, 305, (1960).

[11] CATsch A. Experienta, 13, 312, (1957).

[12] Comar C.L., Bronner F. Eds. "Mineral Metabolism» Academic Press, N.Y. and London (1962).

[13] Comar C.L., Wassermann R.H., Nold M.M. Proc. Soc. Exp. Biol. Med., 92, 859, (1956).

[14] Comar C.L., Wasserman R.H. In « Mineral Metabolism » C.L. Comar, F. Bronner, Eds. Academic Press, N.Y. and London, Vol. 2A, 523, (1964).

[15] Cosmos E. J. Cell. Biol., 23, 241 (1964).

[16] Hammarstrom L., Nilsson A., Ulberg S. Acta Radiol., 63, 183, (1965).

[17] Linkins R.C., Posner A.S., Kunde M.L., Craven D.L. Arch. Biochem. Biophys., $83,472,(1959)$.

[18] LLoYD E. In «Strontium metabolism» J.M.A. Lenihan, J.F. Loutit, J.H. Martin, Eds. 167, Academic Press, N.Y, (1967).

[19] LoNG C. Biochemists Handbook, Spon, London, (1961).

[20] Luning K.G., Frolen H., Nelson A., Ronnback C. Nature, 197, 304, (1963).

[21] Manery J.F., Bale W.F. Amer. J. Physiol., 133, 215, (1941).

[22] Marois M., Morel F. Arch. Anat. Hist. Embr., 34, 275, (1951).

[23] Maynard L.S., Cotzias G.C. J. Biol. Chem., 214, 489, (1955).

[24] Nillsson A., Ulberg S. Acta Radiol., 58, 81, (1962).

[25] Roche J., Michel R., Closon J., Michel O. Biochim. Biophys. Acta, 38, 325, (1960).

[26] SMith H., Bates T.H., UkaEA, P.G. Report 662 (cc), p. 10, (1965).

[27] SPECTOR W.J. «Handbook of Biological Data » Saunders, Philadelphia, (1956).

[28] Spencer H., Lewin I., Samachson J. J. Nucl. Med., 6, 338, (1965).

[29] Thiers R.E., Vallee B.L. J. Biol. Chem., 226, 911, (1957).

[30] TINE J. Rapport technique CRSSA no 10/RC (1972).

[31] TIPTON I.H., CoOK M.J. Health Physics, 9, 103, (1963).

[32] Vasington F.D. Biochim. Biophys. Acta, 113, 414, (1966).

[33] Vaugham J., Williamson M. In "Strontium Metabolism " J.M.A. Lenihan, J.F. Loutit, J.H. Martin, Eds, Academic Press, N.Y., p. 195 (1967).

[34] Weissbuch H., Leporda G., Diaconescu C., Sangereanu O., Cotrau A. In " Contamination par radionucléides ostéotropes et radioprotection » Eds SFRP Paris, Ve congrès int., Grenoble, (France), p. 19, (1971).

[35] Wester P.O. Biochim. Biophys. Acta, 109, 268, (1965).

vol. 9 - $\mathrm{N}^{\circ} 3$ 